Supporting Information for:

Decomposition of A Key Intermediate In Ruthenium Catalyzed Olefin

\title{
Metathesis Reactions
}

Soon Hyeok Hong, Michael W. Day, and Robert H. Grubbs*

Arnold and Mabel Beckman Laboratory of Chemical Synthesis, Division of Chemistry and Chemical Engineering, California Institute of Technology, Pasadena, California 91125

General Considerations. Manipulation of organometallic compounds was performed using standard Schlenk techniques under an atmosphere of dry argon or in a nitrogen-filled Vacuum Atmospheres drybox $\left(\mathrm{O}_{2}<2 \mathrm{ppm}\right)$. NMR spectra were recorded on a Varian Inova $(499.85 \mathrm{MHz}$ for ${ }^{1} \mathrm{H}$; $202.34 \mathrm{MHz}$ for ${ }^{31} \mathrm{P}$; $125.69 \mathrm{MHz}$ for $\left.{ }^{13} \mathrm{C}\right)$ or on a Varian Mercury $300(299.817 \mathrm{MHz}$ for ${ }^{1} \mathrm{H} ; 121.39 \mathrm{MHz}$ for ${ }^{31} \mathrm{P} ; 74.45 \mathrm{MHz}$ for $\left.{ }^{13} \mathrm{C}\right) .{ }^{31} \mathrm{P}$ NMR spectra were referenced using $\mathrm{H}_{3} \mathrm{PO}_{4}(=$ $0 \mathrm{ppm}$ ) as an external standard. Elemental analyses were performed at Desert Analytics (Tucson, AZ). Mass spectra were recorded on JEOL JMS $600 \mathrm{H}$ spectrophotometer. GC spectra were recorded on Hewlett-Packard 5970B MSD with 5890 GC.

Materials and Methods. Benzene, benzene- $\mathrm{d}_{6}$, pentane, and methylene chloride were dried by passage through solvent purification columns. $\mathrm{CD}_{2} \mathrm{Cl}_{2}$ was dried by vacuum transfer from $\mathrm{CaH}_{2}$. All solvents are degassed by standard procedure. Allylbenzene was obtained from Aldrich and used as received. $\left(\mathrm{IMesH}_{2}\right)\left(\mathrm{PCy}_{3}\right)(\mathrm{Cl})_{2} \mathrm{Ru}=\mathrm{CH}_{2}$ was prepared according to literature procedure. ${ }^{2}$

\footnotetext{
${ }^{1}$ Pangborn, A. B.; Giardello, M. A.; Grubbs, R. H.; Rosen, R. K.; Timmers, F. J. Organometallics 1996, 15, 1518-1520.

${ }^{2}$ Sanford, M. S.; Love, J. A.; Grubbs, R. H. J. Am. Chem. Soc. 2001, 123, 6543-6554.
} 
Decomposition of complex 4. Complex $4(48.3 \mathrm{mg}, 0.06 \mathrm{mmol})$ was dissolved in benzene $(2.7 \mathrm{~mL})$ in a sealed tube. The reaction mixture was heated to $55{ }^{\circ} \mathrm{C}$. Precipitation of orange-yellow crystalline solid was observed after $7 \mathrm{hrs}$. After $72 \mathrm{hrs}$, the precipitates were filtered, washed with benzene and dried under vacuum to afford complex $8(13.2 \mathrm{mg}, 46 \%)$. Methyltricyclohexylphosphonium chloride 9 was obtained along with some unidentified decomposed ruthenium species by the addition of pentane $(5 \mathrm{~mL})$ to the filtered benzene solution.

Dinuclear ruthenium complex 8. ${ }^{1} \mathrm{H}$ NMR $\left(\mathrm{CD}_{2} \mathrm{Cl}_{2}\right)$ : 7.14 (s, 1H), 7.05 (s. 1H), 7.04 (s, 1H), $6.90(\mathrm{~s}, 3 \mathrm{H}), 5.62(\mathrm{~s}, 1 \mathrm{H}), 5.44(\mathrm{~s}, 1 \mathrm{H}), 4.19-4.06(\mathrm{~m}, 2 \mathrm{H}), 3.99-3.86(\mathrm{~m}, 6 \mathrm{H}), 2.73$ (br s, 3H), 2.46 (br s, 3H), 2.45 (s, 3H), 2.43 (s, 3H), 2.4-2.3 (br, 9H), 2.27 (s, 3H), 2.17 (s, 3H), 1.96 (br s, 3H), 1.93 (s, 3H), $1.91(\mathrm{~s}, 3 \mathrm{H}),-8.61(\mathrm{~s}, 1 \mathrm{H}) .{ }^{13} \mathrm{C}\left\{{ }^{1} \mathrm{H}\right\} \mathrm{NMR}\left(\mathrm{CD}_{2} \mathrm{Cl}_{2}\right): \quad 413.98,222.73,207.87$, $141.31,140.11,139.64,139.04,138.88,138.86,138.71,138.44,137.68,135.13,134.44,133.65$, $131.88,130.60,129.93,129.89,128.90,128.68,128.28,120.30,111.99,111.85,104.38,100.60$ 98.20, 51.63, 51.37, 48.81, 21.40, 20.99, 20.6-20.5 (br, m), 19.89, 19.25, 19.14, 18.53, 16.95 . Anal. Calcd for $\mathrm{C}_{43} \mathrm{H}_{53} \mathrm{~N}_{4} \mathrm{Cl}_{3} \mathrm{Ru}_{2}$ : C, 55.27; H, 5.72; N, 6.00. Found: C, 55.58; H, 5.64; N, 5.64. HRMS analysis (FAB) m/z: Calcd $\left[\mathrm{M}^{+}\right]$936.1424, found 936.1434.

Methyltricyclohexylphosphonium Chloride 9. ${ }^{1} \mathrm{H} \mathrm{NMR}\left(\mathrm{C}_{6} \mathrm{D}_{6}\right): 2.61\left(\mathrm{~m},\left(\mathrm{CHC}_{5} \mathrm{H}_{10}\right)_{3}-\mathrm{PCH}_{3}{ }^{+}\right.$, $3 \mathrm{H}), 2.42\left(\mathrm{~d}, 3 \mathrm{H}, \mathrm{CH}_{3}-\mathrm{PCy}_{3}{ }^{+}, \mathrm{J}_{\mathrm{HP}}=12.6 \mathrm{~Hz}\right), 1.85-1.00(\mathrm{~m}, 30 \mathrm{H}) .{ }^{13} \mathrm{C}\left\{{ }^{1} \mathrm{H}\right\} \mathrm{NMR}\left(\mathrm{C}_{6} \mathrm{D}_{6}\right): 30.43(\mathrm{~d}$, $\left.\left(\mathrm{CHC}_{5} \mathrm{H}_{10}\right)_{3}-\mathrm{PCH}_{3}^{+}, \mathrm{J}_{\mathrm{CP}}=42.6 \mathrm{~Hz}\right), 27.11\left(\mathrm{~d}, \mathrm{~J}_{\mathrm{CP}}=3.1 \mathrm{~Hz}\right), 26.47\left(\mathrm{~d}, \mathrm{~J}_{\mathrm{CP}}=12.6 \mathrm{~Hz}\right), 25.86,1.5(\mathrm{~d}$, $\left.\mathrm{CH}_{3}-\mathrm{PCy}_{3}{ }^{+}, \mathrm{J}_{\mathrm{CP}}=47.6 \mathrm{~Hz}\right) .{ }^{31} \mathrm{P}\left\{{ }^{1} \mathrm{H}\right\} \mathrm{NMR}\left(\mathrm{C}_{6} \mathrm{D}_{6}\right): 34.5 \mathrm{ppm}$. HRMS analysis (FAB) m/z: Calcd for $\mathrm{C}_{19} \mathrm{H}_{36} \mathrm{P}\left[\mathrm{M}^{+}\right]$: 295.2555, found: 295.2557. 
Figure S1. NOE and ${ }^{1} \mathrm{H}$ NMR spectra of $\mathbf{8}$
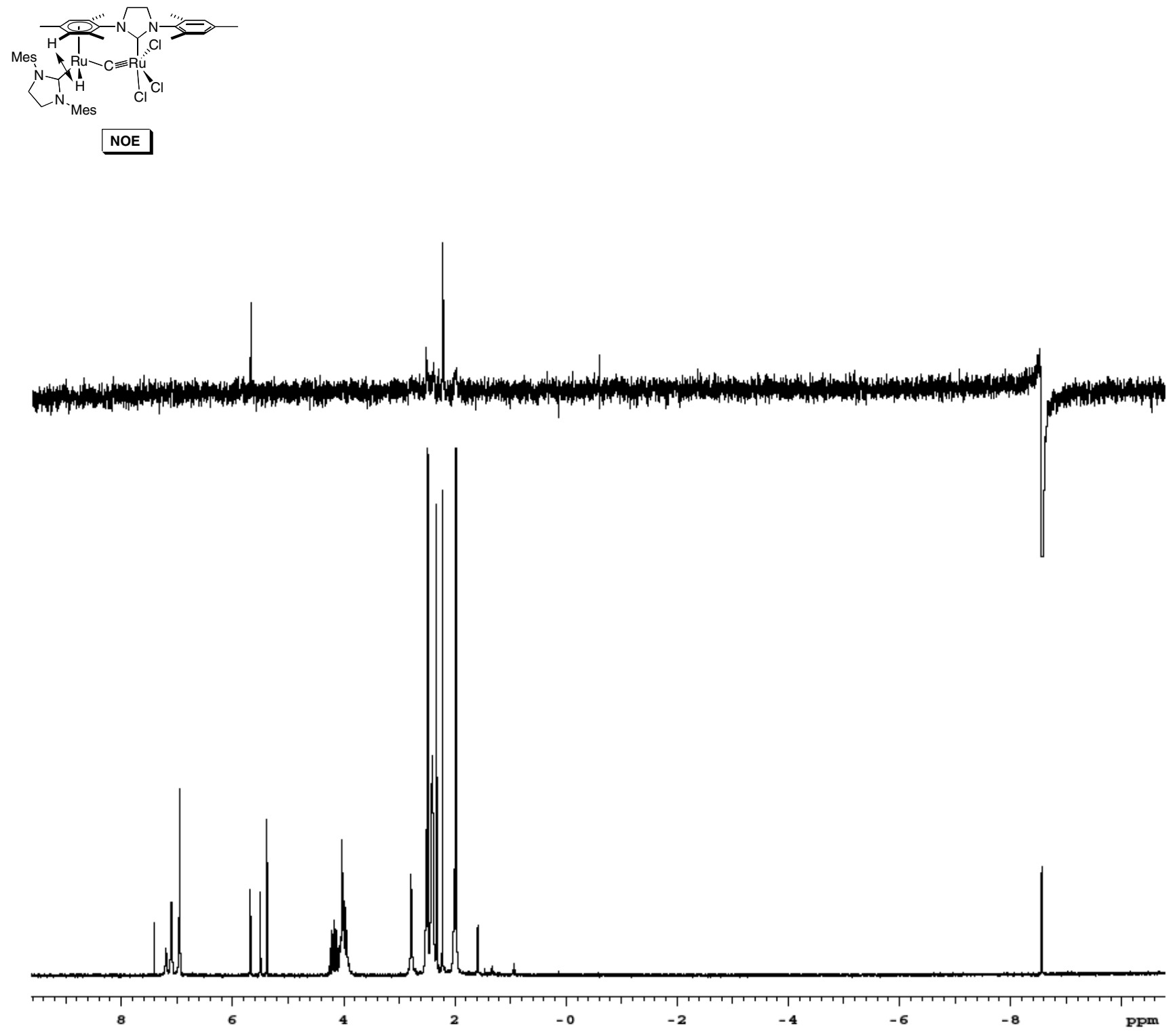
Isomerization reaction of allylbenzene. Allylbenzene $(17.1 \mathrm{mg}, 0.15 \mathrm{mmol})$ and complex 8 (2.0mg, $1.5 \mathrm{~mol} \%)$ were dissolved in $\mathrm{CD}_{2} \mathrm{Cl}_{2}(0.6 \mathrm{~mL})$ in an NMR tube fitted with a screw cap. The resulting solution was heated to $40{ }^{\circ} \mathrm{C}$ and reaction was monitored by measuring the peak heights of allylic protons of allylbenzene and methyl protons of 1-phenyl-1-propene by ${ }^{1} \mathrm{H}$ NMR spectroscopy. After 1 day, yield of 1-phenyl-1-propene was determined by GC (76\%, trans:cis = $8: 1)$. 


\section{Crystal Structure Analysis of Complex 8}

\section{$\underline{\text { Contents }}$}

Table S1. Crystal data

Figures

Table S2. Atomic Coordinates

Table S3. Selected bond distances and angles

Table S4. Full bond distances and angles (for deposit)

Table S5. Anisotropic displacement parameters

Table S6. Observed and calculated structure factors (for deposit)

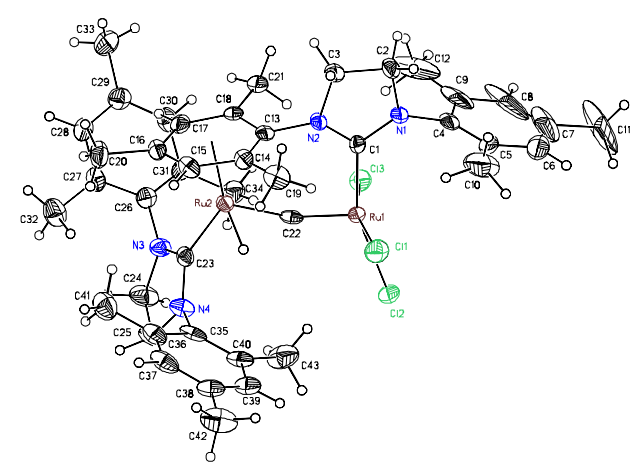

8

Note: Crystallographic data have been deposited at the CCDC, 12 Union Road, Cambridge CB2 1EZ, UK and copies can be obtained on request, free of charge, by quoting the publication citation and the deposition number 223170. 
Table S1. Crystal data and structure refinement for complex 8 (CCDC 223170).

Empirical formula

Formula weight

Crystallization Solvent

Crystal Habit

Crystal size

Crystal color

Type of diffractometer

Wavelength

Data Collection Temperature

$\theta$ range for 10984 reflections used

in lattice determination

Unit cell dimensions

Volume

$\mathrm{Z}$

Crystal system

Space group

Density (calculated)

$\mathrm{F}(000)$

Data collection program

$\theta$ range for data collection

Completeness to $\theta=28.43^{\circ}$

Index ranges

Data collection scan type

Data reduction program

Reflections collected

Independent reflections

Absorption coefficient

Absorption correction

Max. and min. transmission
$\mathrm{C}_{41} \mathrm{H}_{53} \mathrm{Cl}_{3} \mathrm{~N}_{4} \mathrm{Ru} 2 \cdot 1 \frac{1}{2}\left(\mathrm{C}_{6} \mathrm{H}_{6}\right)$

1051.55

Benzene

Needle

$0.38 \times 0.07 \times 0.04 \mathrm{~mm}^{3}$

Yellow/orange

\section{Data Collection}

Rotation

Bruker SMART 1000

$0.71073 \AA \mathrm{MoK} \alpha$

100(2) K

2.39 to $27.68^{\circ}$

$\mathrm{a}=12.4536(9) \AA$

$\mathrm{b}=16.1001(11) \AA$

$\mathrm{c}=24.7739(17) \AA$

$\beta=102.7980(10)^{\circ}$

4843.9(6) $\AA^{3}$

4

Monoclinic

$\mathrm{P} 2{ }_{1} / \mathrm{c}$

$1.442 \mathrm{Mg} / \mathrm{m}^{3}$

2164

Bruker SMART v5.054

1.52 to $28.43^{\circ}$

$92.5 \%$

$-15 \leq \mathrm{h} \leq 16,-20 \leq \mathrm{k} \leq 21,-32 \leq 1 \leq 32$

$\omega$ scans at $3 \phi$ settings

Bruker SAINT v6.022

42525

$11284\left[\mathrm{R}_{\mathrm{int}}=0.0770\right]$

$0.828 \mathrm{~mm}^{-1}$

None

0.9676 and 0.7437 


\section{Table S1 (cont.)}

\section{Structure solution and Refinement}

Structure solution program

Primary solution method

Secondary solution method

Hydrogen placement

Structure refinement program

Refinement method

Data / restraints / parameters

Treatment of hydrogen atoms

Goodness-of-fit on $\mathrm{F}^{2}$

Final $\mathrm{R}$ indices $[\mathrm{I}>2 \sigma(\mathrm{I}), 7073$ reflections]

$\mathrm{R}$ indices (all data)

Type of weighting scheme used

Weighting scheme used

Max shift/error

Average shift/error

Largest diff. peak and hole
SHELXS-97 (Sheldrick, 1990)

Patterson method

Difference Fourier map

Difference Fourier map

SHELXL-97 (Sheldrick, 1997)

Full matrix least-squares on $\mathrm{F}^{2}$

11284 / 0 / 554

Mixed

1.396

$\mathrm{R} 1=0.0488, w \mathrm{R} 2=0.0803$

$\mathrm{R} 1=0.0952, w \mathrm{R} 2=0.0859$

Sigma

$w=1 / \sigma^{2}\left(\mathrm{Fo}^{2}\right)$

0.005

0.000

2.111 and -0.969 e. $\AA^{-3}$

\section{Special Refinement Details}

All hydrogen atoms appear in the Fourier map and were refined as riding atoms with the exception of $\mathrm{H} 2$, a hydride on $\mathrm{Ru} 2$. This hydrogen was refined without restraints. However, care should be taken when interpreting the presence of this hydrogen based solely on the crystallographic data. Hydrides bound to metal atoms are notoriously difficult to resolve with $\mathrm{x}$-ray diffraction data, therefore the results obtained here should be checked for consistency with data from other techniques. There is one residual peak in the final difference Fourier larger than $1 \mathrm{e}-/ \AA^{3}$ and it is impossibly close to a solvent of crystallization.

Refinement of $\mathrm{F}^{2}$ against ALL reflections. The weighted R-factor $(w \mathrm{R})$ and goodness of fit $(\mathrm{S})$ are based on $\mathrm{F}^{2}$, conventional R-factors $(\mathrm{R})$ are based on $\mathrm{F}$, with $\mathrm{F}$ set to zero for negative $\mathrm{F}^{2}$. The threshold expression of $\mathrm{F}^{2}>2 \sigma($ $\mathrm{F}^{2}$ ) is used only for calculating R-factors(gt) etc. and is not relevant to the choice of reflections for refinement. R-factors based on $\mathrm{F}^{2}$ are statistically about twice as large as those based on $\mathrm{F}$, and R-factors based on ALL data will be even larger.

All esds (except the esd in the dihedral angle between two l.s. planes) are estimated using the full covariance matrix. The cell esds are taken into account individually in the estimation of esds in distances, angles and torsion angles; correlations between esds in cell parameters are only used when they are defined by crystal symmetry. An approximate (isotropic) treatment of cell esds is used for estimating esds involving l.s. planes. 


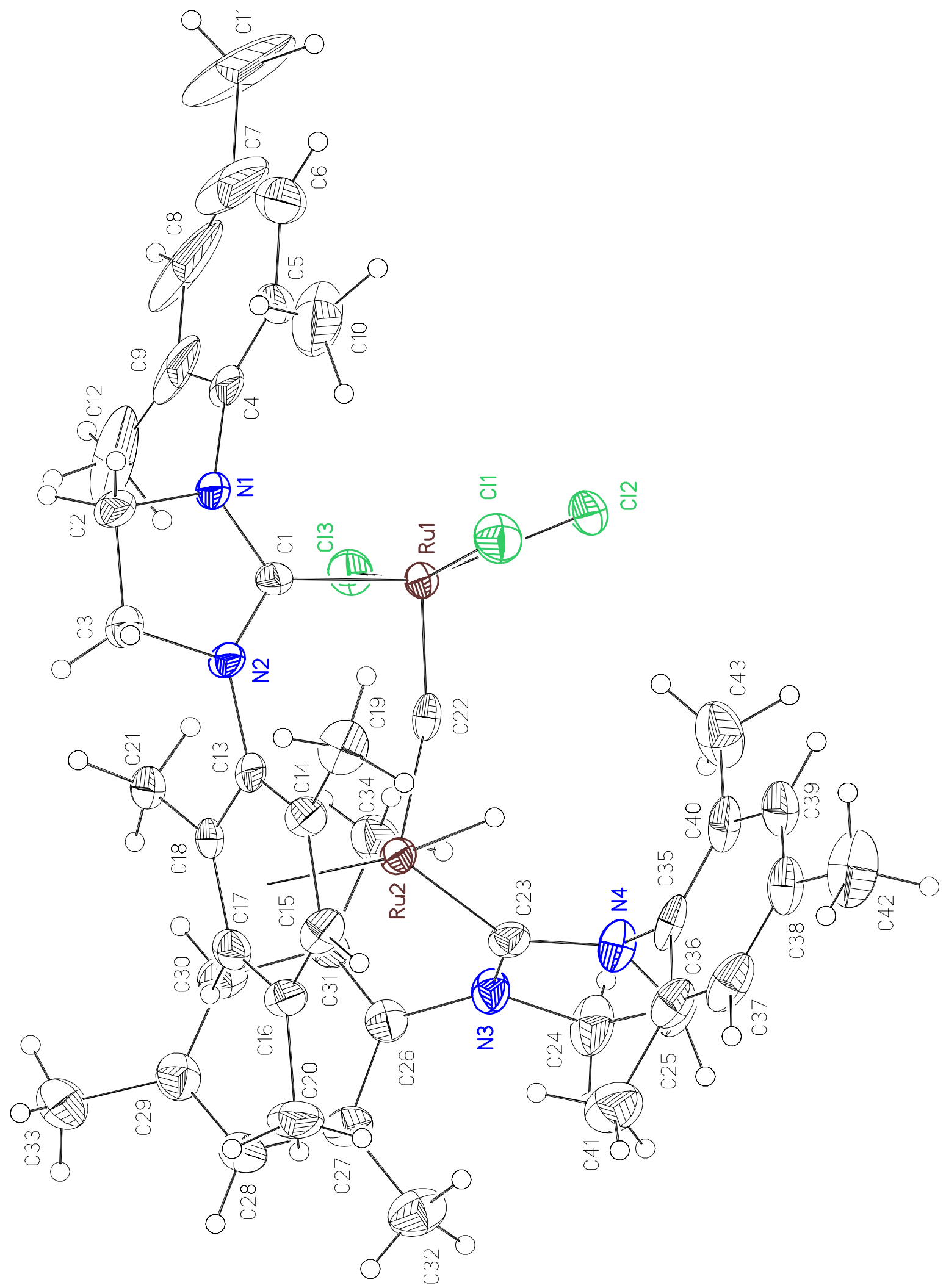

1
$\infty$
1 


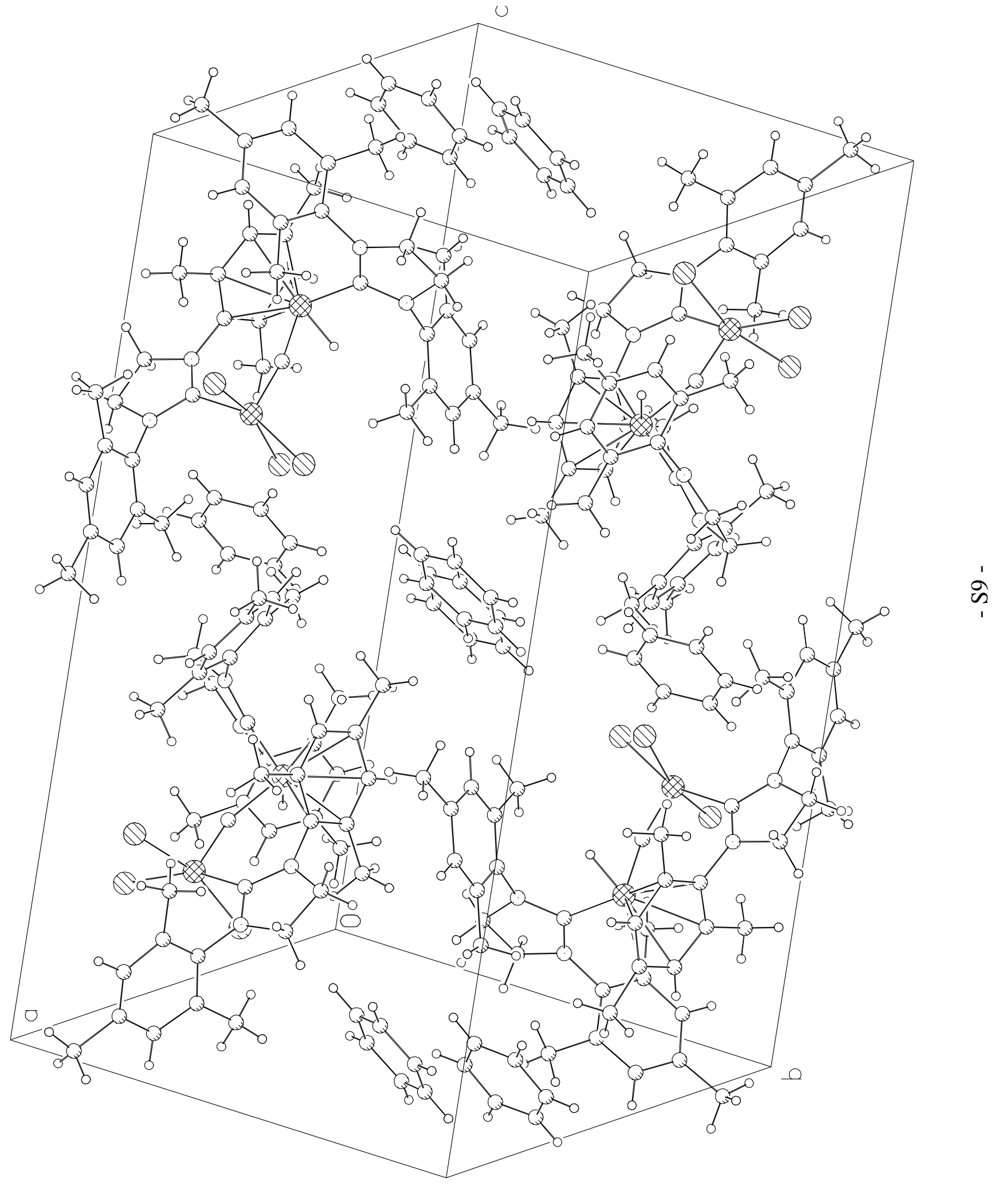



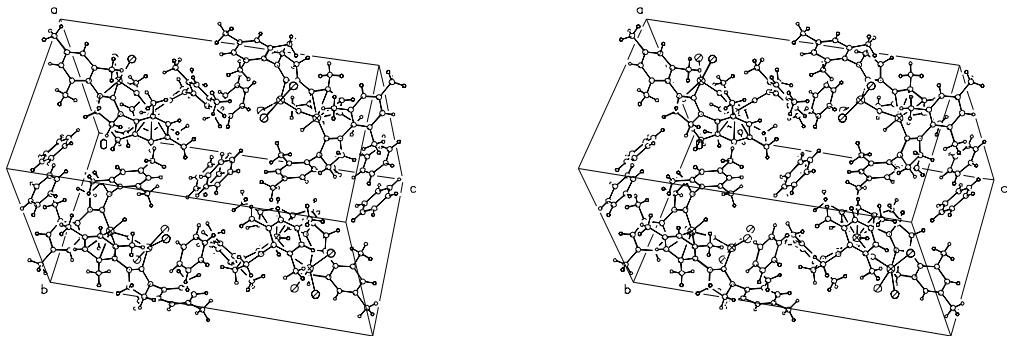

- S10 - 
Table S2. Atomic coordinates $\left(\times 1^{4}\right)$ and equivalent isotropic displacement parameters $\left(\AA^{2} \times 10^{3}\right)$ for $8(C C D C 223170)$. $U(e q)$ is defined as the trace of the orthogonalized $U^{i j}$ tensor.

\begin{tabular}{|c|c|c|c|c|}
\hline & $\mathrm{x}$ & $\mathrm{y}$ & $\mathrm{z}$ & $\mathrm{U}_{\mathrm{eq}}$ \\
\hline $\mathrm{Ru}(1)$ & $8847(1)$ & $2335(1)$ & $7123(1)$ & $22(1)$ \\
\hline $\mathrm{Ru}(2)$ & $7130(1)$ & $1878(1)$ & $8038(1)$ & $22(1)$ \\
\hline $\mathrm{Cl}(1)$ & $7366(1)$ & $2136(1)$ & $6352(1)$ & $36(1)$ \\
\hline $\mathrm{Cl}(2)$ & 8966(1) & $3735(1)$ & $6794(1)$ & $32(1)$ \\
\hline $\mathrm{Cl}(3)$ & $10589(1)$ & $2596(1)$ & $7711(1)$ & $32(1)$ \\
\hline $\mathrm{N}(1)$ & 9591(3) & $635(2)$ & $6887(2)$ & $28(1)$ \\
\hline $\mathrm{N}(2)$ & 8399(3) & $537(2)$ & $7400(1)$ & $22(1)$ \\
\hline $\mathrm{N}(3)$ & $7501(3)$ & $3233(2)$ & $8932(1)$ & $28(1)$ \\
\hline $\mathrm{N}(4)$ & $6234(3)$ & $3588(2)$ & $8219(2)$ & $29(1)$ \\
\hline $\mathrm{C}(1)$ & $8985(3)$ & $1078(2)$ & $7160(2)$ & $21(1)$ \\
\hline $\mathrm{C}(2)$ & $9455(4)$ & $-272(2)$ & $6934(2)$ & $32(1)$ \\
\hline $\mathrm{C}(3)$ & $8621(4)$ & $-345(2)$ & $7291(2)$ & $29(1)$ \\
\hline $\mathrm{C}(4)$ & $10388(5)$ & 929(3) & $6593(2)$ & $48(2)$ \\
\hline $\mathrm{C}(5)$ & $10075(8)$ & $1003(4)$ & $6020(3)$ & $86(3)$ \\
\hline $\mathrm{C}(6)$ & 10933(10) & $1261(5)$ & $5756(4)$ & 141(6) \\
\hline $\mathrm{C}(7)$ & $12002(13)$ & $1354(6)$ & $6062(7)$ & $178(9)$ \\
\hline $\mathrm{C}(8)$ & $12236(7)$ & $1252(4)$ & $6594(5)$ & $137(5)$ \\
\hline $\mathrm{C}(9)$ & $11472(6)$ & 1016(3) & $6895(4)$ & $71(2)$ \\
\hline$C(10)$ & 8955(8) & $827(4)$ & $5699(3)$ & $124(4)$ \\
\hline $\mathrm{C}(11)$ & $12919(11)$ & $1624(4)$ & $5757(6)$ & $331(11)$ \\
\hline $\mathrm{C}(12)$ & $11786(5)$ & $832(3)$ & $7496(3)$ & $88(2)$ \\
\hline $\mathrm{C}(13)$ & 7764(4) & $686(2)$ & $7796(2)$ & $22(1)$ \\
\hline $\mathrm{C}(14)$ & $6605(4)$ & $677(2)$ & $7624(2)$ & $26(1)$ \\
\hline $\mathrm{C}(15)$ & $5988(4)$ & $772(2)$ & $8038(2)$ & $29(1)$ \\
\hline$C(16)$ & $6496(4)$ & $872(2)$ & $8596(2)$ & $29(1)$ \\
\hline $\mathrm{C}(17)$ & $7663(4)$ & $889(2)$ & $8749(2)$ & $28(1)$ \\
\hline $\mathrm{C}(18)$ & $8318(3)$ & $769(2)$ & $8365(2)$ & $23(1)$ \\
\hline C(19) & $6040(4)$ & $542(3)$ & $7032(2)$ & $37(1)$ \\
\hline $\mathrm{C}(20)$ & $5817(4)$ & $895(3)$ & $9029(2)$ & $43(1)$ \\
\hline $\mathrm{C}(21)$ & $9538(3)$ & 739(2) & $8524(2)$ & $29(1)$ \\
\hline $\mathrm{C}(22)$ & $8059(3)$ & $2296(2)$ & $7601(2)$ & $22(1)$ \\
\hline $\mathrm{C}(23)$ & $6927(3)$ & $2975(2)$ & $8434(2)$ & $25(1)$ \\
\hline $\mathrm{C}(24)$ & $7289(4)$ & $4117(3)$ & $9048(2)$ & $43(1)$ \\
\hline$C(25)$ & $6299(4)$ & $4315(3)$ & $8586(2)$ & $38(1)$ \\
\hline$C(26)$ & 8193(4) & $2733(3)$ & $9345(2)$ & $27(1)$ \\
\hline $\mathrm{C}(27)$ & $7739(4)$ & $2402(3)$ & $9769(2)$ & $30(1)$ \\
\hline $\mathrm{C}(28)$ & $8395(4)$ & $1866(3)$ & $10149(2)$ & $33(1)$ \\
\hline C(29) & $9455(4)$ & $1663(3)$ & $10110(2)$ & $33(1)$ \\
\hline $\mathrm{C}(30)$ & $9898(4)$ & $2058(3)$ & $9714(2)$ & $33(1)$ \\
\hline $\mathrm{C}(31)$ & $9289(4)$ & $2613(3)$ & $9333(2)$ & $28(1)$ \\
\hline $\mathrm{C}(32)$ & $6613(4)$ & $2634(3)$ & $9840(2)$ & $44(1)$ \\
\hline $\mathrm{C}(33)$ & $10096(4)$ & $1024(3)$ & 10491(2) & $46(1)$ \\
\hline $\mathrm{C}(34)$ & $9802(4)$ & $3053(3)$ & 8919(2) & $39(1)$ \\
\hline$C(35)$ & $5311(3)$ & $3526(2)$ & $7756(2)$ & $28(1)$ \\
\hline$C(36)$ & $4318(4)$ & $3213(3)$ & $7844(2)$ & $33(1)$ \\
\hline $\mathrm{C}(37)$ & $3419(4)$ & $3203(3)$ & $7402(2)$ & $39(1)$ \\
\hline $\mathrm{C}(38)$ & $3472(4)$ & $3497(3)$ & $6884(2)$ & $35(1)$ \\
\hline C(39) & $4458(4)$ & $3788(3)$ & $6812(2)$ & $37(1)$ \\
\hline
\end{tabular}




\begin{tabular}{lrrrr}
$\mathrm{C}(40)$ & $5392(4)$ & $3817(2)$ & $7237(2)$ & $32(1)$ \\
$\mathrm{C}(41)$ & $4222(4)$ & $2881(3)$ & $8390(2)$ & $48(1)$ \\
$\mathrm{C}(42)$ & $2457(4)$ & $3525(3)$ & $6422(2)$ & $57(2)$ \\
$\mathrm{C}(43)$ & $6462(4)$ & $4128(3)$ & $7126(2)$ & $53(2)$ \\
& & & \\
$\mathrm{C}(51)$ & $3888(5)$ & $861(4)$ & $9939(3)$ & $68(2)$ \\
$\mathrm{C}(52)$ & $3294(5)$ & $309(4)$ & $9557(3)$ & $75(2)$ \\
$\mathrm{C}(53)$ & $2542(4)$ & $603(4)$ & $9101(3)$ & $63(2)$ \\
$\mathrm{C}(54)$ & $2400(4)$ & $1455(4)$ & $9034(2)$ & $59(2)$ \\
$\mathrm{C}(55)$ & $2972(4)$ & $1979(4)$ & $9418(2)$ & $54(2)$ \\
$\mathrm{C}(56)$ & $3719(5)$ & $1682(4)$ & $9876(3)$ & $60(2)$ \\
$\mathrm{C}(61)$ & & & \\
$\mathrm{C}(62)$ & $4649(7)$ & $4204(4)$ & $-47(4)$ & $77(2)$ \\
$\mathrm{C}(63)$ & $5441(7)$ & $4419(4)$ & $389(3)$ & $69(2)$ \\
& $5818(5)$ & $5224(6)$ & $439(3)$ & $86(2)$ \\
\hline
\end{tabular}


Table S3. Selected bond lengths $[\AA]$ and angles $\left[^{\circ}\right]$ for complex 8 (CCDC 223170).

\begin{tabular}{lclc}
\hline $\mathrm{Ru}(1)-\mathrm{C}(22)$ & $1.698(4)$ & $\mathrm{C}(22)-\mathrm{Ru}(1)-\mathrm{C}(1)$ & $89.29(17)$ \\
$\mathrm{Ru}(1)-\mathrm{C}(1)$ & $2.031(4)$ & $\mathrm{C}(22)-\mathrm{Ru}(1)-\mathrm{Cl}(1)$ & $95.19(13)$ \\
$\mathrm{Ru}(1)-\mathrm{Cl}(1)$ & $2.3653(12)$ & $\mathrm{C}(1)-\mathrm{Ru}(1)-\mathrm{Cl}(1)$ & $86.80(12)$ \\
$\mathrm{Ru}(1)-\mathrm{Cl}(3)$ & $2.3669(11)$ & $\mathrm{C}(22)-\mathrm{Ru}(1)-\mathrm{Cl}(3)$ & $99.79(13)$ \\
$\mathrm{Ru}(1)-\mathrm{Cl}(2)$ & $2.4124(11)$ & $\mathrm{C}(1)-\mathrm{Ru}(1)-\mathrm{Cl}(3)$ & $95.29(12)$ \\
$\mathrm{Ru}(2)-\mathrm{H}(2)$ & $1.45(3)$ & $\mathrm{Cl}(1)-\operatorname{Ru}(1)-\mathrm{Cl}(3)$ & $164.89(4)$ \\
$\mathrm{Ru}(2)-\mathrm{C}(22)$ & $1.875(4)$ & $\mathrm{C}(22)-\mathrm{Ru}(1)-\mathrm{Cl}(2)$ & $110.97(13)$ \\
$\mathrm{Ru}(2)-\mathrm{C}(23)$ & $2.063(4)$ & $\mathrm{C}(1)-\mathrm{Ru}(1)-\mathrm{Cl}(2)$ & $159.29(12)$ \\
& & $\mathrm{Cl}(1)-\operatorname{Ru}(1)-\mathrm{Cl}(2)$ & $87.09(4)$ \\
& & $\mathrm{Cl}(3)-\operatorname{Ru}(1)-\mathrm{Cl}(2)$ & $85.74(4)$ \\
& & $\mathrm{H}(2)-\operatorname{Ru}(2)-\mathrm{C}(22)$ & $74.1(13)$ \\
& & $\mathrm{H}(2)-\operatorname{Ru}(2)-\mathrm{C}(23)$ & $83.9(13)$ \\
& & $\mathrm{C}(22)-\mathrm{Ru}(2)-\mathrm{C}(23)$ & $97.12(16)$ \\
& & $\mathrm{Ru}(1)-\mathrm{C}(22)-\mathrm{Ru}(2)$ & $160.3(2)$ \\
\hline
\end{tabular}


Table S4. Bond lengths [ $[\AA]$ and angles $\left[{ }^{\circ}\right]$ for complex 8 (CCDC 223170).

\begin{tabular}{|c|c|c|c|}
\hline $\mathrm{Ru}(1)-\mathrm{C}(22)$ & $1.698(4)$ & $C(29)-C(30)$ & $1.382(6)$ \\
\hline $\mathrm{Ru}(1)-\mathrm{C}(1)$ & $2.031(4)$ & $C(29)-C(33)$ & $1.501(6)$ \\
\hline $\mathrm{Ru}(1)-\mathrm{Cl}(1)$ & $2.3653(12)$ & $\mathrm{C}(30)-\mathrm{C}(31)$ & $1.396(6)$ \\
\hline $\mathrm{Ru}(1)-\mathrm{Cl}(3)$ & $2.3669(11)$ & $\mathrm{C}(31)-\mathrm{C}(34)$ & $1.502(6)$ \\
\hline $\mathrm{Ru}(1)-\mathrm{Cl}(2)$ & $2.4124(11)$ & $C(35)-C(40)$ & $1.394(6)$ \\
\hline $\mathrm{Ru}(2)-\mathrm{H}(2)$ & $1.45(3)$ & $C(35)-C(36)$ & $1.396(6)$ \\
\hline $\mathrm{Ru}(2)-\mathrm{C}(22)$ & $1.875(4)$ & $\mathrm{C}(36)-\mathrm{C}(37)$ & $1.382(6)$ \\
\hline $\mathrm{Ru}(2)-\mathrm{C}(23)$ & $2.063(4)$ & $\mathrm{C}(36)-\mathrm{C}(41)$ & $1.483(6)$ \\
\hline $\mathrm{Ru}(2)-\mathrm{C}(13)$ & $2.207(4)$ & $\mathrm{C}(37)-\mathrm{C}(38)$ & $1.384(6)$ \\
\hline $\mathrm{Ru}(2)-\mathrm{C}(14)$ & $2.219(4)$ & $\mathrm{C}(38)-\mathrm{C}(39)$ & $1.362(6)$ \\
\hline $\mathrm{Ru}(2)-\mathrm{C}(15)$ & $2.280(4)$ & $\mathrm{C}(38)-\mathrm{C}(42)$ & $1.506(6)$ \\
\hline $\mathrm{Ru}(2)-\mathrm{C}(18)$ & $2.345(4)$ & $\mathrm{C}(39)-\mathrm{C}(40)$ & $1.385(6)$ \\
\hline $\mathrm{Ru}(2)-\mathrm{C}(17)$ & $2.357(4)$ & $\mathrm{C}(40)-\mathrm{C}(43)$ & $1.503(6)$ \\
\hline $\mathrm{Ru}(2)-\mathrm{C}(16)$ & $2.375(4)$ & $C(51)-C(56)$ & $1.343(7)$ \\
\hline $\mathrm{N}(1)-\mathrm{C}(1)$ & $1.328(5)$ & $\mathrm{C}(51)-\mathrm{C}(52)$ & $1.387(8)$ \\
\hline $\mathrm{N}(1)-\mathrm{C}(4)$ & $1.435(6)$ & $\mathrm{C}(52)-\mathrm{C}(53)$ & $1.382(8)$ \\
\hline $\mathrm{N}(1)-\mathrm{C}(2)$ & $1.478(5)$ & $C(53)-C(54)$ & $1.389(7)$ \\
\hline $\mathrm{N}(2)-\mathrm{C}(1)$ & $1.357(5)$ & $C(54)-C(55)$ & $1.351(7)$ \\
\hline $\mathrm{N}(2)-\mathrm{C}(13)$ & $1.411(5)$ & $C(55)-C(56)$ & $1.382(7)$ \\
\hline $\mathrm{N}(2)-\mathrm{C}(3)$ & $1.483(5)$ & $C(61)-C(62)$ & $1.337(8)$ \\
\hline $\mathrm{N}(3)-\mathrm{C}(23)$ & $1.346(5)$ & $C(61)-C(63) \# 1$ & $1.371(8)$ \\
\hline $\mathrm{N}(3)-\mathrm{C}(26)$ & $1.432(5)$ & C(62)-C(63) & $1.376(8)$ \\
\hline $\mathrm{N}(3)-\mathrm{C}(24)$ & $1.487(5)$ & $\mathrm{C}(63)-\mathrm{C}(61) \# 1$ & $1.371(8)$ \\
\hline $\mathrm{N}(4)-\mathrm{C}(23)$ & $1.341(5)$ & & \\
\hline $\mathrm{N}(4)-\mathrm{C}(35)$ & $1.437(5)$ & $\mathrm{C}(22)-\mathrm{Ru}(1)-\mathrm{C}(1)$ & $89.29(17)$ \\
\hline $\mathrm{N}(4)-\mathrm{C}(25)$ & $1.472(5)$ & $\mathrm{C}(22)-\mathrm{Ru}(1)-\mathrm{Cl}(1)$ & $95.19(13)$ \\
\hline $\mathrm{C}(2)-\mathrm{C}(3)$ & $1.510(5)$ & $\mathrm{C}(1)-\mathrm{Ru}(1)-\mathrm{Cl}(1)$ & $86.80(12)$ \\
\hline$C(4)-C(5)$ & $1.391(8)$ & $\mathrm{C}(22)-\mathrm{Ru}(1)-\mathrm{Cl}(3)$ & $99.79(13)$ \\
\hline$C(4)-C(9)$ & $1.398(8)$ & $\mathrm{C}(1)-\mathrm{Ru}(1)-\mathrm{Cl}(3)$ & $95.29(12)$ \\
\hline$C(5)-C(6)$ & $1.434(11)$ & $\mathrm{Cl}(1)-\mathrm{Ru}(1)-\mathrm{Cl}(3)$ & $164.89(4)$ \\
\hline$C(5)-C(10)$ & $1.473(10)$ & $\mathrm{C}(22)-\mathrm{Ru}(1)-\mathrm{Cl}(2)$ & $110.97(13)$ \\
\hline$C(6)-C(7)$ & $1.386(19)$ & $\mathrm{C}(1)-\mathrm{Ru}(1)-\mathrm{Cl}(2)$ & $159.29(12)$ \\
\hline $\mathrm{C}(7)-\mathrm{C}(8)$ & $1.296(17)$ & $\mathrm{Cl}(1)-\mathrm{Ru}(1)-\mathrm{Cl}(2)$ & $87.09(4)$ \\
\hline$C(7)-C(11)$ & $1.565(12)$ & $\mathrm{Cl}(3)-\mathrm{Ru}(1)-\mathrm{Cl}(2)$ & $85.74(4)$ \\
\hline $\mathrm{C}(8)-\mathrm{C}(9)$ & $1.385(9)$ & $\mathrm{H}(2)-\mathrm{Ru}(2)-\mathrm{C}(22)$ & 74.1(13) \\
\hline C(9)-C(12) & $1.484(9)$ & $\mathrm{H}(2)-\mathrm{Ru}(2)-\mathrm{C}(23)$ & $83.9(13)$ \\
\hline$C(13)-C(14)$ & $1.411(6)$ & $C(22)-R u(2)-C(23)$ & 97.12(16) \\
\hline$C(13)-C(18)$ & $1.430(6)$ & $\mathrm{H}(2)-\mathrm{Ru}(2)-\mathrm{C}(13)$ & $110.6(13)$ \\
\hline$C(14)-C(15)$ & $1.420(6)$ & $C(22)-R u(2)-C(13)$ & 81.52(16) \\
\hline$C(14)-C(19)$ & $1.496(6)$ & $C(23)-R u(2)-C(13)$ & $164.18(17)$ \\
\hline$C(15)-C(16)$ & $1.394(6)$ & $\mathrm{H}(2)-\mathrm{Ru}(2)-\mathrm{C}(14)$ & 86.6(13) \\
\hline$C(16)-C(17)$ & $1.419(6)$ & $\mathrm{C}(22)-\mathrm{Ru}(2)-\mathrm{C}(14)$ & 101.67(16) \\
\hline$C(16)-C(20)$ & $1.506(6)$ & $C(23)-R u(2)-C(14)$ & $155.68(16)$ \\
\hline $\mathrm{C}(17)-\mathrm{C}(18)$ & $1.396(5)$ & $\mathrm{C}(13)-\mathrm{Ru}(2)-\mathrm{C}(14)$ & $37.19(15)$ \\
\hline$C(18)-C(21)$ & $1.484(5)$ & $\mathrm{H}(2)-\mathrm{Ru}(2)-\mathrm{C}(15)$ & 93.9(13) \\
\hline$C(24)-C(25)$ & $1.520(6)$ & $\mathrm{C}(22)-\mathrm{Ru}(2)-\mathrm{C}(15)$ & $138.18(16)$ \\
\hline$C(26)-C(31)$ & $1.385(5)$ & $C(23)-R u(2)-C(15)$ & $121.74(15)$ \\
\hline$C(26)-C(27)$ & $1.402(6)$ & $C(13)-R u(2)-C(15)$ & $65.25(15)$ \\
\hline$C(27)-C(28)$ & $1.400(6)$ & $\mathrm{C}(14)-\mathrm{Ru}(2)-\mathrm{C}(15)$ & $36.78(14)$ \\
\hline$C(27)-C(32)$ & $1.499(6)$ & $\mathrm{H}(2)-\mathrm{Ru}(2)-\mathrm{C}(18)$ & $147.0(13)$ \\
\hline$C(28)-C(29)$ & $1.384(6)$ & $\mathrm{C}(22)-\mathrm{Ru}(2)-\mathrm{C}(18)$ & $92.88(15)$ \\
\hline
\end{tabular}




\begin{tabular}{|c|c|c|c|}
\hline$C(23)-R u(2)-C(18)$ & $128.46(16)$ & $\mathrm{N}(2)-\mathrm{C}(13)-\mathrm{Ru}(2)$ & $128.7(3)$ \\
\hline $\mathrm{C}(13)-\mathrm{Ru}(2)-\mathrm{C}(18)$ & $36.47(14)$ & $\mathrm{C}(18)-\mathrm{C}(13)-\mathrm{Ru}(2)$ & $77.0(2)$ \\
\hline $\mathrm{C}(14)-\mathrm{Ru}(2)-\mathrm{C}(18)$ & $66.10(15)$ & $C(13)-C(14)-C(15)$ & $117.4(4)$ \\
\hline $\mathrm{C}(15)-\mathrm{Ru}(2)-\mathrm{C}(18)$ & $75.57(15)$ & $C(13)-C(14)-C(19)$ & $121.7(4)$ \\
\hline $\mathrm{H}(2)-\mathrm{Ru}(2)-\mathrm{C}(17)$ & $156.6(13)$ & $C(15)-C(14)-C(19)$ & $120.8(4)$ \\
\hline $\mathrm{C}(22)-\mathrm{Ru}(2)-\mathrm{C}(17)$ & $124.48(16)$ & $C(13)-C(14)-R u(2)$ & $71.0(2)$ \\
\hline $\mathrm{C}(23)-\mathrm{Ru}(2)-\mathrm{C}(17)$ & $105.47(16)$ & $\mathrm{C}(15)-\mathrm{C}(14)-\mathrm{Ru}(2)$ & $73.9(2)$ \\
\hline $\mathrm{C}(13)-\mathrm{Ru}(2)-\mathrm{C}(17)$ & $63.70(15)$ & $C(19)-C(14)-R u(2)$ & $127.3(3)$ \\
\hline $\mathrm{C}(14)-\mathrm{Ru}(2)-\mathrm{C}(17)$ & $76.23(15)$ & $C(16)-C(15)-C(14)$ & $121.9(4)$ \\
\hline $\mathrm{C}(15)-\mathrm{Ru}(2)-\mathrm{C}(17)$ & $62.89(16)$ & $C(16)-C(15)-R u(2)$ & $76.3(2)$ \\
\hline $\mathrm{C}(18)-\mathrm{Ru}(2)-\mathrm{C}(17)$ & $34.55(13)$ & $\mathrm{C}(14)-\mathrm{C}(15)-\mathrm{Ru}(2)$ & $69.3(2)$ \\
\hline $\mathrm{H}(2)-\mathrm{Ru}(2)-\mathrm{C}(16)$ & $122.8(13)$ & $C(15)-C(16)-C(17)$ & $118.7(4)$ \\
\hline$C(22)-R u(2)-C(16)$ & $155.20(16)$ & $C(15)-C(16)-C(20)$ & $120.4(4)$ \\
\hline$C(23)-R u(2)-C(16)$ & $102.43(15)$ & $C(17)-C(16)-C(20)$ & $120.8(4)$ \\
\hline $\mathrm{C}(13)-\mathrm{Ru}(2)-\mathrm{C}(16)$ & $75.50(15)$ & $C(15)-C(16)-R u(2)$ & $68.9(2)$ \\
\hline $\mathrm{C}(14)-\mathrm{Ru}(2)-\mathrm{C}(16)$ & $64.68(15)$ & $C(17)-C(16)-R u(2)$ & $71.9(2)$ \\
\hline $\mathrm{C}(15)-\mathrm{Ru}(2)-\mathrm{C}(16)$ & $34.78(14)$ & $C(20)-C(16)-R u(2)$ & $135.2(3)$ \\
\hline$C(18)-R u(2)-C(16)$ & $62.97(15)$ & $C(18)-C(17)-C(16)$ & $122.3(4)$ \\
\hline $\mathrm{C}(17)-\mathrm{Ru}(2)-\mathrm{C}(16)$ & $34.89(14)$ & $\mathrm{C}(18)-\mathrm{C}(17)-\mathrm{Ru}(2)$ & $72.2(2)$ \\
\hline $\mathrm{C}(1)-\mathrm{N}(1)-\mathrm{C}(4)$ & $128.1(3)$ & $C(16)-C(17)-R u(2)$ & $73.2(2)$ \\
\hline $\mathrm{C}(1)-\mathrm{N}(1)-\mathrm{C}(2)$ & $113.8(3)$ & $C(17)-C(18)-C(13)$ & $117.1(4)$ \\
\hline $\mathrm{C}(4)-\mathrm{N}(1)-\mathrm{C}(2)$ & $118.0(3)$ & $\mathrm{C}(17)-\mathrm{C}(18)-\mathrm{C}(21)$ & $122.8(4)$ \\
\hline $\mathrm{C}(1)-\mathrm{N}(2)-\mathrm{C}(13)$ & 129.4(3) & $C(13)-C(18)-C(21)$ & $120.0(4)$ \\
\hline $\mathrm{C}(1)-\mathrm{N}(2)-\mathrm{C}(3)$ & $113.2(3)$ & $C(17)-C(18)-R u(2)$ & $73.2(2)$ \\
\hline $\mathrm{C}(13)-\mathrm{N}(2)-\mathrm{C}(3)$ & $116.6(3)$ & $C(13)-C(18)-R u(2)$ & $66.5(2)$ \\
\hline $\mathrm{C}(23)-\mathrm{N}(3)-\mathrm{C}(26)$ & $126.5(3)$ & $C(21)-C(18)-R u(2)$ & $130.4(3)$ \\
\hline $\mathrm{C}(23)-\mathrm{N}(3)-\mathrm{C}(24)$ & 113.1(3) & $\mathrm{Ru}(1)-\mathrm{C}(22)-\mathrm{Ru}(2)$ & $160.3(2)$ \\
\hline $\mathrm{C}(26)-\mathrm{N}(3)-\mathrm{C}(24)$ & $120.2(3)$ & N(4)-C(23)-N(3) & $107.5(4)$ \\
\hline C(23)-N(4)-C(35) & $126.6(3)$ & $\mathrm{N}(4)-\mathrm{C}(23)-\mathrm{Ru}(2)$ & $125.3(3)$ \\
\hline $\mathrm{C}(23)-\mathrm{N}(4)-\mathrm{C}(25)$ & $113.5(3)$ & $\mathrm{N}(3)-\mathrm{C}(23)-\mathrm{Ru}(2)$ & 127.1(3) \\
\hline $\mathrm{C}(35)-\mathrm{N}(4)-\mathrm{C}(25)$ & $118.2(3)$ & $\mathrm{N}(3)-\mathrm{C}(24)-\mathrm{C}(25)$ & $101.7(3)$ \\
\hline $\mathrm{N}(1)-\mathrm{C}(1)-\mathrm{N}(2)$ & 107.4(3) & $\mathrm{N}(4)-\mathrm{C}(25)-\mathrm{C}(24)$ & $102.8(3)$ \\
\hline N(1)-C(1)-Ru(1) & $124.4(3)$ & $C(31)-C(26)-C(27)$ & $121.7(4)$ \\
\hline $\mathrm{N}(2)-\mathrm{C}(1)-\mathrm{Ru}(1)$ & $127.8(3)$ & $\mathrm{C}(31)-\mathrm{C}(26)-\mathrm{N}(3)$ & $120.2(4)$ \\
\hline $\mathrm{N}(1)-\mathrm{C}(2)-\mathrm{C}(3)$ & $103.2(3)$ & $\mathrm{C}(27)-\mathrm{C}(26)-\mathrm{N}(3)$ & $118.1(4)$ \\
\hline $\mathrm{N}(2)-\mathrm{C}(3)-\mathrm{C}(2)$ & $102.3(3)$ & $C(28)-C(27)-C(26)$ & $117.8(4)$ \\
\hline$C(5)-C(4)-C(9)$ & $123.8(6)$ & $C(28)-C(27)-C(32)$ & $119.7(4)$ \\
\hline$C(5)-C(4)-N(1)$ & $118.5(6)$ & $C(26)-C(27)-C(32)$ & $122.4(4)$ \\
\hline $\mathrm{C}(9)-\mathrm{C}(4)-\mathrm{N}(1)$ & 117.2(5) & $\mathrm{C}(29)-\mathrm{C}(28)-\mathrm{C}(27)$ & $121.4(4)$ \\
\hline C(4)-C(5)-C(6) & $115.0(9)$ & $C(30)-C(29)-C(28)$ & $118.5(4)$ \\
\hline$C(4)-C(5)-C(10)$ & $123.5(6)$ & $C(30)-C(29)-C(33)$ & $121.5(4)$ \\
\hline$C(6)-C(5)-C(10)$ & $121.5(8)$ & $\mathrm{C}(28)-\mathrm{C}(29)-\mathrm{C}(33)$ & $120.0(4)$ \\
\hline$C(7)-C(6)-C(5)$ & $120.2(11)$ & $C(29)-C(30)-C(31)$ & $122.1(4)$ \\
\hline C(8)-C(7)-C(6) & $121.0(11)$ & $C(26)-C(31)-C(30)$ & $117.7(4)$ \\
\hline $\mathrm{C}(8)-\mathrm{C}(7)-\mathrm{C}(11)$ & $120.2(16)$ & $C(26)-C(31)-C(34)$ & $121.3(4)$ \\
\hline$C(6)-C(7)-C(11)$ & 118.7(14) & $\mathrm{C}(30)-\mathrm{C}(31)-\mathrm{C}(34)$ & $120.9(4)$ \\
\hline $\mathrm{C}(7)-\mathrm{C}(8)-\mathrm{C}(9)$ & $123.7(12)$ & $\mathrm{C}(40)-\mathrm{C}(35)-\mathrm{C}(36)$ & $120.9(4)$ \\
\hline C(8)-C(9)-C(4) & $115.9(8)$ & $\mathrm{C}(40)-\mathrm{C}(35)-\mathrm{N}(4)$ & $120.3(4)$ \\
\hline C(8)-C(9)-C(12) & $122.4(8)$ & $\mathrm{C}(36)-\mathrm{C}(35)-\mathrm{N}(4)$ & $118.6(4)$ \\
\hline $\mathrm{C}(4)-\mathrm{C}(9)-\mathrm{C}(12)$ & $121.7(5)$ & $C(37)-C(36)-C(35)$ & $118.0(5)$ \\
\hline $\mathrm{C}(14)-\mathrm{C}(13)-\mathrm{N}(2)$ & $118.7(4)$ & $\mathrm{C}(37)-\mathrm{C}(36)-\mathrm{C}(41)$ & $120.2(4)$ \\
\hline$C(14)-C(13)-C(18)$ & $122.5(4)$ & $C(35)-C(36)-C(41)$ & $121.7(4)$ \\
\hline N(2)-C(13)-C(18) & $118.6(4)$ & $\mathrm{C}(38)-\mathrm{C}(37)-\mathrm{C}(36)$ & $122.3(4)$ \\
\hline$C(14)-C(13)-R u(2)$ & $71.8(2)$ & $\mathrm{C}(39)-\mathrm{C}(38)-\mathrm{C}(37)$ & $117.9(4)$ \\
\hline
\end{tabular}




$\begin{array}{llll}\mathrm{C}(39)-\mathrm{C}(38)-\mathrm{C}(42) & 121.3(5) & \mathrm{C}(52)-\mathrm{C}(53)-\mathrm{C}(54) & 118.7(6) \\ \mathrm{C}(37)-\mathrm{C}(38)-\mathrm{C}(42) & 120.7(5) & \mathrm{C}(55)-\mathrm{C}(54)-\mathrm{C}(53) & 120.0(6) \\ \mathrm{C}(38)-\mathrm{C}(39)-\mathrm{C}(40) & 122.9(5) & \mathrm{C}(54)-\mathrm{C}(55)-\mathrm{C}(56) & 121.1(6) \\ \mathrm{C}(39)-\mathrm{C}(40)-\mathrm{C}(35) & 117.9(4) & \mathrm{C}(51)-\mathrm{C}(56)-\mathrm{C}(55) & 119.7(6) \\ \mathrm{C}(39)-\mathrm{C}(40)-\mathrm{C}(43) & 120.2(5) & \mathrm{C}(62)-\mathrm{C}(61)-\mathrm{C}(63) \# 1 & 121.3(6) \\ \mathrm{C}(35)-\mathrm{C}(40)-\mathrm{C}(43) & 121.9(4) & \mathrm{C}(61)-\mathrm{C}(62)-\mathrm{C}(63) & 119.6(6) \\ \mathrm{C}(56)-\mathrm{C}(51)-\mathrm{C}(52) & 120.3(6) & \mathrm{C}(62)-\mathrm{C}(63)-\mathrm{C}(61) \# 1 & 119.1(6) \\ \mathrm{C}(53)-\mathrm{C}(52)-\mathrm{C}(51) & 120.1(6) & & \end{array}$

Symmetry transformations used to generate equivalent atoms:

\#1 -x+1,-y+1,-z 
Table S5. Anisotropic displacement parameters $\left(\AA^{2} \times 10^{4}\right)$ for complex 8 (CCDC 223170). The anisotropic displacement factor exponent takes the form: $-2 \pi^{2}\left[h^{2} a^{* 2} U^{11}+\ldots+2 h\right.$ k a* b* $^{* 12}$ ]

\begin{tabular}{|c|c|c|c|c|c|c|}
\hline & $\mathrm{U}^{11}$ & $\mathrm{U}^{22}$ & $\mathrm{U}^{33}$ & $\mathrm{U}^{23}$ & $\mathrm{U}^{13}$ & $\mathrm{U}^{12}$ \\
\hline $\mathrm{Ru}(1)$ & $257(2)$ & $162(2)$ & 251(2) & $26(2)$ & $99(2)$ & $14(2)$ \\
\hline $\mathrm{Ru}(2)$ & $232(2)$ & $168(2)$ & 283(2) & $3(2)$ & $104(2)$ & $-7(2)$ \\
\hline $\mathrm{Cl}(1)$ & $428(7)$ & $360(7)$ & $269(7)$ & $-25(5)$ & 14(5) & $10(5)$ \\
\hline $\mathrm{Cl}(2)$ & $436(7)$ & 191(6) & $378(7)$ & $60(5)$ & $164(6)$ & $7(5)$ \\
\hline $\mathrm{Cl}(3)$ & $248(6)$ & $314(6)$ & $402(7)$ & $57(5)$ & $91(5)$ & $-23(5)$ \\
\hline $\mathrm{N}(1)$ & $370(20)$ & 165(19) & $350(20)$ & $47(17)$ & 195(19) & $58(17)$ \\
\hline $\mathrm{N}(2)$ & $280(20)$ & $132(18)$ & $280(20)$ & $-26(16)$ & 136(17) & $-6(16)$ \\
\hline $\mathrm{N}(3)$ & $350(20)$ & $210(20)$ & $270(20)$ & $-36(17)$ & $45(18)$ & $38(17)$ \\
\hline $\mathrm{N}(4)$ & $250(20)$ & $220(20)$ & $360(20)$ & $-42(18)$ & $-14(18)$ & $22(17)$ \\
\hline$C(1)$ & $260(30)$ & $220(20)$ & $180(20)$ & $-12(19)$ & 69(19) & 11(19) \\
\hline$C(2)$ & $430(30)$ & $200(20)$ & 390(30) & $-20(20)$ & $240(20)$ & $20(20)$ \\
\hline$C(3)$ & $390(30)$ & $150(20)$ & $370(30)$ & $-10(20)$ & $170(20)$ & $10(20)$ \\
\hline C(4) & $720(40)$ & 130(30) & 780(40) & 170(30) & $570(40)$ & $180(30)$ \\
\hline $\mathrm{C}(5)$ & $1720(80)$ & $420(40)$ & $770(50)$ & $380(40)$ & $940(60)$ & $620(50)$ \\
\hline$C(6)$ & $2940(150)$ & $510(50)$ & $1350(90)$ & $560(50)$ & $1700(110)$ & $950(90)$ \\
\hline$C(7)$ & $2960(170)$ & $290(50)$ & $3100(200)$ & $510(80)$ & $2770(180)$ & $600(90)$ \\
\hline$C(8)$ & $1060(70)$ & $160(40)$ & $3430(160)$ & $-90(60)$ & $1650(100)$ & $10(40)$ \\
\hline $\mathrm{C}(9)$ & $640(50)$ & $90(30)$ & $1640(70)$ & $40(40)$ & $730(50)$ & $80(30)$ \\
\hline$C(10)$ & $2250(110)$ & $1160(60)$ & $330(40)$ & $100(40)$ & $300(50)$ & $1210(70)$ \\
\hline $\mathrm{C}(11)$ & $5100(200)$ & $460(50)$ & $6400(200)$ & $740(90)$ & $5600(200)$ & $590(80)$ \\
\hline$C(12)$ & $420(40)$ & $360(40)$ & $1690(80)$ & $-100(40)$ & $-100(40)$ & $120(30)$ \\
\hline $\mathrm{C}(13)$ & $300(30)$ & $70(20)$ & $310(30)$ & $-7(18)$ & $110(20)$ & 2(18) \\
\hline$C(14)$ & $250(30)$ & $200(20)$ & $360(30)$ & $-10(20)$ & $120(20)$ & $-62(19)$ \\
\hline$C(15)$ & $220(30)$ & $200(20)$ & $500(30)$ & $-50(20)$ & $150(20)$ & $-76(19)$ \\
\hline$C(16)$ & $380(30)$ & $140(20)$ & $410(30)$ & $-20(20)$ & $240(20)$ & $-60(20)$ \\
\hline$C(17)$ & $400(30)$ & $180(20)$ & $270(30)$ & 12(19) & $120(20)$ & $-10(20)$ \\
\hline $\mathrm{C}(18)$ & $310(30)$ & $110(20)$ & $280(30)$ & $41(18)$ & $90(20)$ & 41(19) \\
\hline C(19) & 290(30) & $320(30)$ & $460(30)$ & $-70(20)$ & $20(20)$ & $-90(20)$ \\
\hline$C(20)$ & $590(40)$ & $340(30)$ & $480(30)$ & $-30(20)$ & $360(30)$ & $-90(30)$ \\
\hline $\mathrm{C}(21)$ & $370(30)$ & $200(20)$ & $320(30)$ & $30(20)$ & $110(20)$ & $60(20)$ \\
\hline $\mathrm{C}(22)$ & $220(20)$ & $100(20)$ & $310(30)$ & $-22(19)$ & $-6(19)$ & $13(18)$ \\
\hline $\mathrm{C}(23)$ & $230(20)$ & $230(20)$ & $310(30)$ & $0(20)$ & $130(20)$ & $-31(19)$ \\
\hline $\mathrm{C}(24)$ & $550(40)$ & $260(30)$ & $430(30)$ & $-110(20)$ & $10(30)$ & $70(20)$ \\
\hline$C(25)$ & $300(30)$ & $260(30)$ & $530(30)$ & $-110(20)$ & $-10(20)$ & $70(20)$ \\
\hline$C(26)$ & $310(30)$ & $260(20)$ & $210(20)$ & $-50(20)$ & $20(20)$ & $-20(20)$ \\
\hline $\mathrm{C}(27)$ & $290(30)$ & $320(30)$ & $310(30)$ & $-70(20)$ & $110(20)$ & $-40(20)$ \\
\hline $\mathrm{C}(28)$ & $370(30)$ & $370(30)$ & $280(30)$ & $20(20)$ & $110(20)$ & $-20(20)$ \\
\hline C(29) & $340(30)$ & $350(30)$ & $270(30)$ & $-10(20)$ & $10(20)$ & $10(20)$ \\
\hline $\mathrm{C}(30)$ & $260(30)$ & $440(30)$ & $310(30)$ & $-40(20)$ & $60(20)$ & $0(20)$ \\
\hline $\mathrm{C}(31)$ & $330(30)$ & 270(30) & $250(30)$ & $30(20)$ & $70(20)$ & $-30(20)$ \\
\hline $\mathrm{C}(32)$ & $320(30)$ & $590(30)$ & $400(30)$ & $-90(30)$ & $90(20)$ & $0(30)$ \\
\hline $\mathrm{C}(33)$ & $500(40)$ & $540(30)$ & $310(30)$ & $90(30)$ & $50(30)$ & $70(30)$ \\
\hline $\mathrm{C}(34)$ & $340(30)$ & $390(30)$ & $460(30)$ & $80(20)$ & $110(20)$ & $-50(20)$ \\
\hline $\mathrm{C}(35)$ & $180(30)$ & $150(20)$ & $470(30)$ & $-90(20)$ & $-20(20)$ & $47(19)$ \\
\hline$C(36)$ & $280(30)$ & $250(30)$ & $460(30)$ & $-100(20)$ & $90(20)$ & $10(20)$ \\
\hline $\mathrm{C}(37)$ & 190(30) & $300(30)$ & $680(40)$ & $-120(30)$ & $80(30)$ & $-10(20)$ \\
\hline $\mathrm{C}(38)$ & $350(30)$ & $190(20)$ & $460(30)$ & $0(20)$ & $-10(20)$ & $20(20)$ \\
\hline
\end{tabular}




\begin{tabular}{lrrrrrr}
$\mathrm{C}(39)$ & $420(30)$ & $220(30)$ & $470(30)$ & $50(20)$ & $70(30)$ & $50(20)$ \\
$\mathrm{C}(40)$ & $230(30)$ & $170(20)$ & $530(30)$ & $40(20)$ & $30(20)$ & $30(20)$ \\
$\mathrm{C}(41)$ & $400(30)$ & $510(40)$ & $570(40)$ & $-40(30)$ & $200(30)$ & $-20(30)$ \\
$\mathrm{C}(42)$ & $500(40)$ & $370(30)$ & $700(40)$ & $-140(30)$ & $-140(30)$ & $10(30)$ \\
$\mathrm{C}(43)$ & $340(30)$ & $460(30)$ & $780(40)$ & $240(30)$ & $80(30)$ & $-40(30)$ \\
& & & & & & \\
$\mathrm{C}(51)$ & $560(40)$ & $860(50)$ & $590(40)$ & $290(40)$ & $60(30)$ & $-40(40)$ \\
$\mathrm{C}(52)$ & $550(40)$ & $620(40)$ & $1130(60)$ & $370(40)$ & $280(40)$ & $30(40)$ \\
$\mathrm{C}(53)$ & $340(40)$ & $800(50)$ & $740(50)$ & $60(40)$ & $110(30)$ & $-90(30)$ \\
$\mathrm{C}(54)$ & $320(30)$ & $770(50)$ & $630(40)$ & $200(40)$ & $50(30)$ & $-40(30)$ \\
$\mathrm{C}(55)$ & $340(30)$ & $550(40)$ & $750(50)$ & $40(30)$ & $160(30)$ & $-50(30)$ \\
$\mathrm{C}(56)$ & $490(40)$ & $770(50)$ & $600(40)$ & $10(40)$ & $250(30)$ & $10(30)$ \\
& & & & & & \\
$\mathrm{C}(61)$ & $910(60)$ & $570(50)$ & $900(60)$ & $70(50)$ & $340(50)$ & $30(40)$ \\
$\mathrm{C}(62)$ & $1100(60)$ & $600(50)$ & $460(40)$ & $80(30)$ & $340(40)$ & $390(40)$ \\
$\mathrm{C}(63)$ & $560(50)$ & $1170(70)$ & $810(60)$ & $-340(50)$ & $90(40)$ & $250(50)$ \\
\hline
\end{tabular}

